\title{
Von Daten zu Stochastischen Modellen
}

\author{
Peter Bühlmann
}

Peter Bühlmann wurde 1965 in Zürich geboren. Er studierte an der ETH Zürich, wo er 1993 promovierte. Danach war er für ein Jahr als Research Fellow und dann für zwei Jahre als Neyman Assistant Professor am Department of Statistics der University of California in Berkeley tätig. Seit 1997 ist er Assistenzprofessor an der ETH Zürich. Seine Forschungsinteressen liegen in der Statistik und der Wahrscheinlichkeitstheorie, vorwiegend für Anwendungen bei abhängigen Daten; dazu kommen auch Gebiete der Informationstheorie und des "Computing". Sein von Haus aus miteingeprägtes Hobby ist das Bergsteigen, das er heute mit Vorliebe mit seiner Ehefrau und in abgeschwächter Form auch bereits mit seinen beiden Töchtern ausübt.

\section{Einleitung}

Ein wohl allen empirischen Wissenschaften gemeinsames Ziel ist das Schliessen von Daten der realen Welt auf abstrakte Modelle. Ein Modell ist strikte genommen einfach eine Menge, welche mit Verknüpfungsregeln für deren Elemente versehen ist. In den Anwendungen wird dann dessen Interpretation, welche eine allgemeine vereinfachende Beschreibung des Beobachteten liefert, oder dessen Prognose-Potenzial wichtig sein. Wir beschränken uns hier auf stochastische Modelle.

Der Vorgang, wie man von Daten auf stochastische Modelle schliessen kann, gehört zum Kern der Statistik. Er ist massgebend unterstützt von der induktiven Logik: da das

Das Problem, aus Messdaten die "wahre" Struktur herauszulesen oder auf Grund von Messdaten Aussagen über das zukünftige Verhalten zu machen, ist für jede empirische Wissenschaft grundlegend. Im allgemeinen bedingt diese Aufgabe, ein mathematisches Modell zu erarbeiten und auszuwählen, das in der Lage ist, den Vorgang möglichst genau zu beschreiben. Je nach der ursprünglichen Fragestellung gibt es dabei möglicherweise verschiedene "beste" Modelle. Für den Prozess der Modellwahl stellt die heutige Stochastik eine Reihe von mächtigen Hilfsmitteln bereit. In seinem Beitrag illustriert Peter Bühlmann einige davon an konkreten Beispielen aus den unterschiedlichsten Gebieten: Wasserstand des Rio Negro, Helligkeit eines "White Dwarf" Sternes, DNA von Drosophila, tägliche Returns von Aktien. Es sind dies gleichzeitig Beispiele für innovative mathematische Anwendungen in einer beeindruckenden Vielzahl von Umgebungen. ust 
Schliessen von endlich vielen Beobachtungen (dem Besonderen) auf ein Modell (dem Allgemeinen) nicht mit Sicherheit möglich ist, benützt man die Wahrscheinlichkeitstheorie, um mit gewissen (typischerweise grossen) Wahrscheinlichkeiten immer noch Aussagen über das Allgemeine zu machen. Wir werden auf diese grundlagentheoretischen Aspekte nicht näher eingehen. Auch ist der Prozess "von Daten zu stochastischen Modellen" häufig von einer interessierenden Fragestellung beeinflusst. Noch bevor man zu den Daten kommt, sollte idealerweise die Fragestellung im Zentrum stehen: zuerst Fragestellung, dann Daten und schliesslich stochastische Modell-Bildung. Wir werden aber auch diesen ersten Schritt, welcher zum Beispiel die Planung eines Experimentes beinhaltet, nicht weiter diskutieren.

Vielmehr möchten wir, vorwiegend exemplarisch, einen kleinen Aspekt der stochastischen Modell-Bildung diskutieren: er beinhaltet eine sehr beschränkte Auswahl von Problemstellungen. Das statistische Testen von Hypothesen wird kurz angeschnitten, hauptsächlich wird aber auf das in gewissem Sinne komplementäre Problem von optimalen Vorhersage-Modellen eingegangen. Dabei streifen wir Methoden der quantitativen Bestrafung für komplexe Modelle, Optimalität bei vielfältigen, riesigen Modell-Klassen und ausblickend einen modernen Ansatz der Mittelung von komplexen Modellen. Alle realen Daten-Beispiele handeln von zeitlich abhängigen Beobachtungen.

\section{Zwei Ansätze für Modellwahl}

Verschiedenste Methoden für die Wahl eines stochastischen Modells können vom Verwendungszweck her grundsätzlich in zwei Klassen eingeteilt werden: der strukturelle Ansatz, wo die Struktur eines Modells interessiert, oder der entscheidungstheoretische Ansatz, welcher als Ziel ein optimales Vorhersage-Potenzial des Modells hat. Wie wir sehen werden, kann der entscheidungstheoretische Ansatz auch interessante strukturelle Informationen liefern, umgekehrt erhält man aber im allgemeinen mit dem strukturellen Ansatz keine vollständige Information für eine optimale Prognose.

2.1 Struktureller Ansatz. Es geht hier darum, signifikante (oder populärer ausgedrückt: relevante) Struktur oder zumindest einige signifikante strukturelle Komponenten der Daten zu entdecken. Dafür benützen wir den Formalismus des statistischen Tests, welcher auf einem Falsifizierungs-Argument beruht: es kann bloss eine (Null-)Hypothese probabilistisch verworfen, aber nicht bewiesen werden. Einer (Null-)Hypothese ist immer eine Alternative entgegengesetzt. Dieser Formalismus kann folgendermassen konkretisiert werden. Als Grundlage ist ein allgemeines Basis-Modell spezifiziert, so dass die (Null-)Hypothese ein Spezialfall dieses allgemeinen Basis-Modells ist. Die Alternative ist dann das Komplement der (Null-)Hypothese bezüglich des Basis-Modells.

Das folgende einfache "Spielzeug-Problem" illustriert den grundlegenden Gedanken bei der strukturellen Modellwahl, welcher auch bei realen Anwendungen in viel komplizierteren Situationen prägend ist. Abbildung 1 zeigt $n=100$ simulierte Daten $\left(x_{1}, Y_{1}\right)$, $\ldots,\left(x_{n}, Y_{n}\right)$. Es scheint vernünftig, als Basis-Modell ein einfaches lineares RegressionsModell anzunehmen,

$$
Y_{i}=\beta_{0}+\beta_{1} x_{i}+\varepsilon_{i}(i=1, \ldots, n),
$$

wobei $\varepsilon_{1}, \ldots, \varepsilon_{n}$ unabhängig und identisch verteilt (i.i.d.) sind mit Erwartungswert $\mathbb{E}\left[\varepsilon_{i}\right]=0$ und $\operatorname{Varianz} \operatorname{Var}\left(\varepsilon_{i}\right)=\sigma^{2}<\infty$. Als Nullhypothese $\left(H_{0}\right)$ betrachten wir 


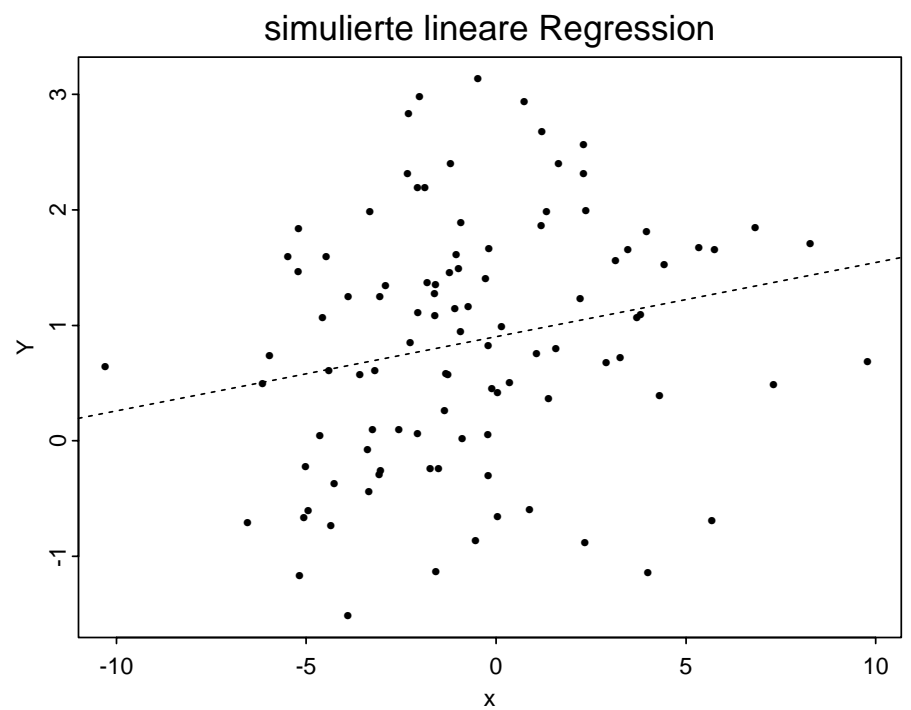

Abb. 1100 simulierte Datenpunkte einer einfachen linearen Regression mit geschätzter Regressionsgerade (Kleinste-Quadrate Schätzung).

den Spezialfall des Basis-Modells,

$$
H_{0}: \beta_{1}=0
$$

welcher besagt, dass es keinen Einfluss von erklärenden Variablen $x_{i}$ auf $Y_{i}(i=1, \ldots, n)$ gibt. Die Frage ist jetzt, ob die Nullhypothese $H_{0}$ oder das Basis-Modell mit $\beta_{1} \neq$ 0 adäquater zur Beschreibung der Daten ist. Rein visuell, siehe Abbildung 1, kommt man zu keinem klaren, eindeutigen Schluss. Auch nicht mit der Punktschätzung $\hat{\beta}_{1}=$ $0.064 \neq 0$ für die Steigung der Regressionsgeraden, da diese Schätzung bloss wegen der zufälligen Rauschterme $\varepsilon_{i}(i=1, \ldots, n)$ von Null verschieden sein könnte. Mit Hilfe des klassischen $t$-Tests findet man aufgrund der Daten, dass die Nullhypothese auf dem 5\% Test-Niveau ${ }^{1}$ ) verworfen wird. Der wahre Wert, welchen wir hier ja bei diesem simulierten Beispiel kennen, ist $\beta_{1}=0.05$, und der statistische $t$-Test entscheidet also bei diesen Daten richtig.

Wir möchten im Folgenden kurz auf ein in der Praxis interessierendes reales DatenBeispiel eingehen, wo ein viel komplizierteres strukturelles Problem vorliegt.

Beispiel 1: Wasserstand des Rio Negro in Manaus (Brasilien).

Die Daten bestehen aus täglichen Messungen von 1903-1992, welche insgesamt 32874 Werte ergeben. Natürlich weisen diese starke saisonale Schwankungen auf, welche hier nicht primär interessieren. In Abbildung 2 sind deshalb korrigierte Wasserstandswerte gegeben, so dass die Saison-Effekte verschwinden sollten, siehe Brillinger (1997). Die

1) Das heisst, die Wahrscheinlichkeit für einen Fehler 1. Art ist 5\%. Ein Fehler 1. Art bedeutet, dass die Nullhypothese fälschlicherweise vom statistischen Test verworfen wird. 


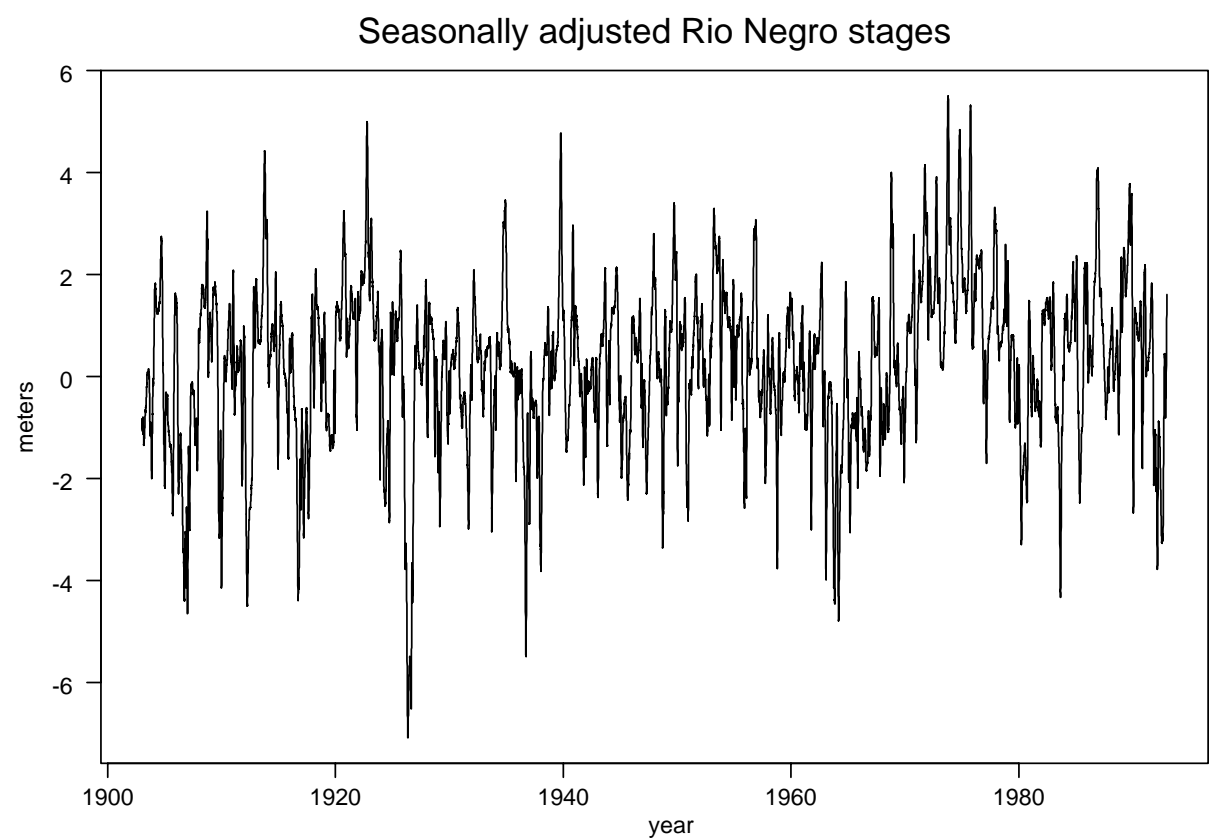

Abb. 2 Saison-korrigierte Wasserstände des Rio Negro, zentriert um Null.

uns interessierende Fragestellung lautet hier: Gibt es eine systematische Erhöhung des Wasserstandes im Verlaufe der Zeit? Diese Fragestellung wird oft im Zusammenhang mit der Abholzung im Einzugsgebiet des Rio Negro diskutiert, da ein Baum mit seinen Wurzeln ein natürliches Aufsauge-Potenzial für Regenwasser besitzt.

Als allgemeines stochastisches Basis-Modell betrachten wir

$$
X_{t}=m_{t}+\varepsilon_{t}(t=1, \ldots, n=32874),
$$

wobei $X_{t}$ den Saison-korrigierten Wasserstand, $m_{t}$ einen deterministischen Trend und $\varepsilon_{t}$ einen Rausch-Term zum Zeitpunkt $t$ bezeichnen. Spezifischer nehmen wir an, dass $\left(m_{t}\right)_{t}$ eine schwach monoton wachsende Trend-Folge ist und $\left(\varepsilon_{t}\right)_{t}$ farbige stationäre Rausch-Terme darstellen, d.h. alle mit Erwartungswert $\mathbb{E}\left[\varepsilon_{t}\right]=0$, aber im Gegensatz zu weissem Rauschen sind $\varepsilon_{s}$ und $\varepsilon_{t}$ korreliert für $s \neq t$. Die Annahme von farbigem Rauschen ist von den Daten her motiviert, die eine Zeitreihe bilden. Die ursprünglich interessierende Fragestellung, übersetzt in die Sprache der Modell-Welt, kann wie im obigen "Spielzeug-Problem" mit einer Nullhypothese formalisiert werden: $m_{t} \equiv m$ für alle $t$. Aus Gründen einer vernünftigen Asymptotik zum Testen dieser Nullhypothese, betrachtet man anstelle einer Folge indiziert mit $\mathbb{N}$ ein reskaliertes Kurven-Problem. Bei Stichprobengrösse $n$ sei $m_{t}=m(t / n)(t=1, \ldots, n)$, wobei $m():.[0,1] \rightarrow \mathbb{R}$ eine schwach monoton wachsende Kurve ist. Bei zunehmendem $n$ beobachtet man die Kurve $m($.$) also an immer dichter liegenden Punkten. Als Nullhypothese formulieren wir dann,$

$$
H_{0}: m(x) \equiv m \text { für alle } x \in[0,1] .
$$

welche ein Untermodell des allgemeinen Basis-Modells darstellt. 
Fitted monotonic and 19 replicates

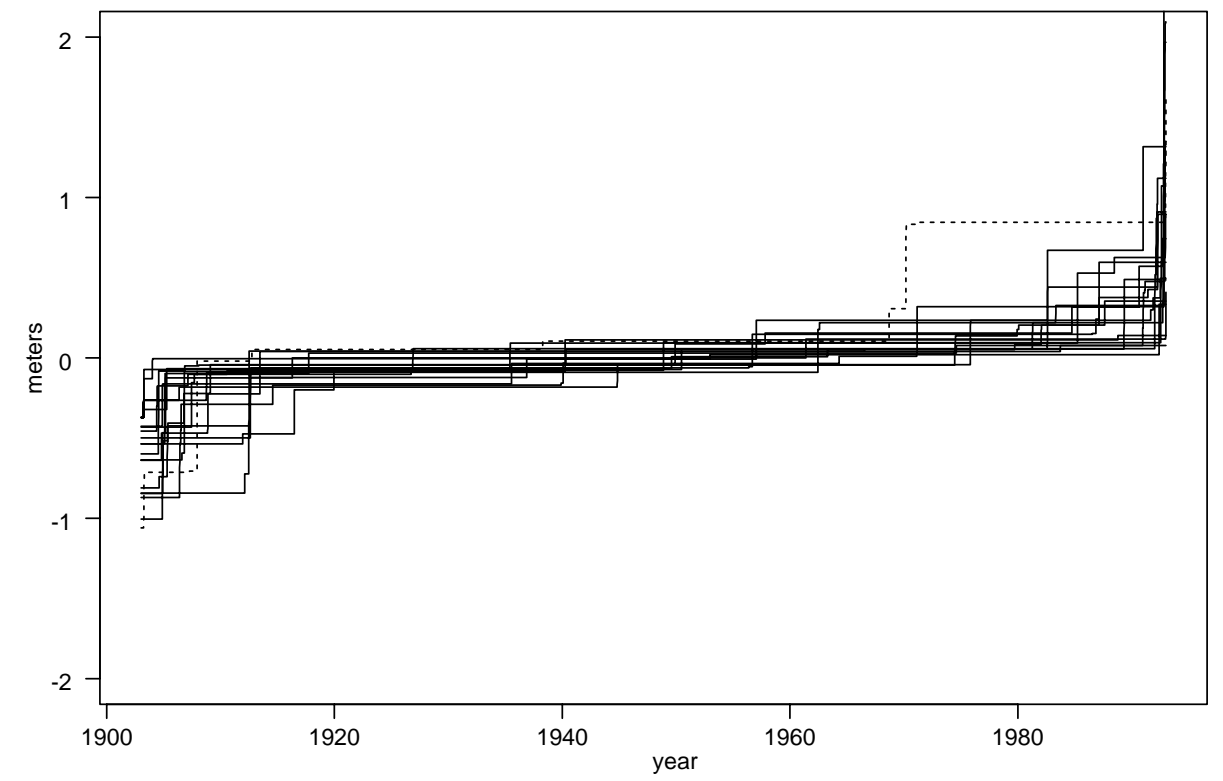

Abb. 3 Geschätzte Trendkurve (gepunktet) und 19 Replikate dieser Schätzung unter $H_{0}$ (ausgezogen), zentriert um Null.

Aufgrund der Daten kann diese Nullhypothese getestet werden, obschon wir es mit einem schwierigen, nichtparametrischen Problem zu tun haben: die Rausch-Terme sind farbig (abhängig) und die Trend-Kurve $m($.$) ist unendlich-dimensional. Die folgenden$ gefundenen Resultate sind von Brillinger (1997). Abbildung 3 zeigt eine Schätzung $\hat{m}($. der Trend-Kurve $m($.$) , basierend auf dem "Pool-Adjacent-Violator"-Algorithmus, siehe$ Friedman \& Tibshirani (1984). Die Frage ist dann, ob sich die Sprünge in der Schätzung, wo also die Trend-Kurve strikt monoton wächst, bloss aufgrund der Rausch-Effekte zeigen. Um dies zu beantworten werden 19 Replikate dieser Schätzung unter der Nullhypothese $H_{0}$ erzeugt. Dafür wird im zentrierten Fall $m_{t} \equiv 0$ gesetzt; die Schwierigkeit liegt dann in einer geeigneten Simulation des unbekannten Prozesses $\left(\varepsilon_{t}\right)_{t}$, welche mit einer Resampling-Technik durchgeführt wird. Man kann zeigen, dass so erhaltene Replikate des Kurven-Schätzers die statistische Variation von $\hat{m}($.$) asymptotisch korrekt$ beschreiben, falls die Nullhypothese stimmt. Schätzer und Replikate ergeben zusammen 20 Kurven; auf dem 5\% Test-Niveau fragt man, ob die geschätzte Kurve an irgendeiner Stelle die Extremste unter allen zwanzig ist. Gemäss Abbildung 3 ist dies der Fall und wir schliessen, dass $H_{0}$ verworfen wird. Brillinger (1997) beschreibt das Resultat als "there is a soupçon of an increasing trend".

Wir hatten es hier also mit einem Beispiel zu tun, wo es um die "wahre" Struktur eines Modells geht. Der Begriff "Wahrheit" ist dabei selbstverständlich bloss bezüglich eines postulierten allgemeinen Basis-Modells zu verstehen. 


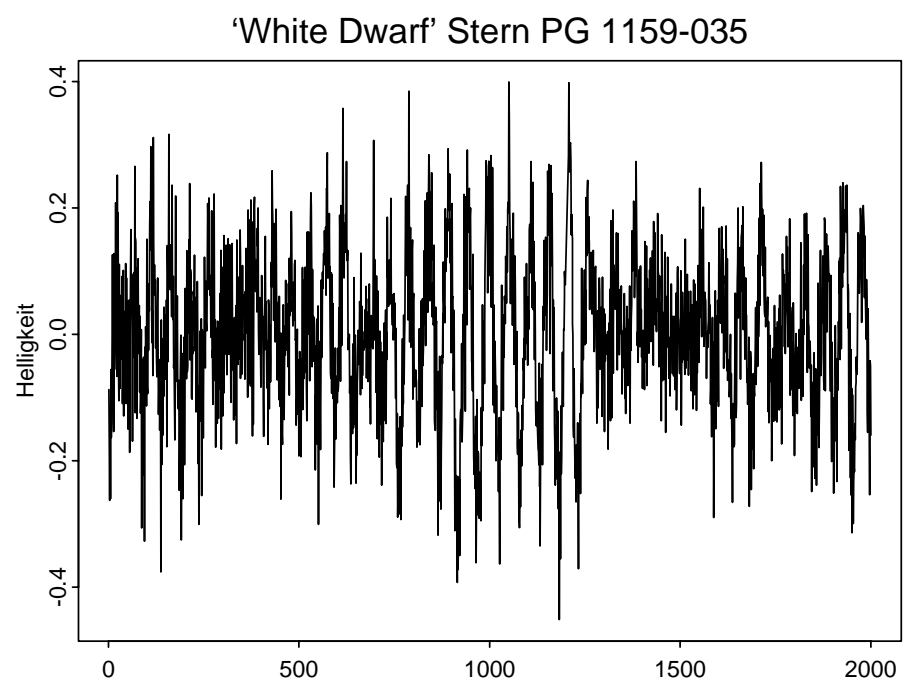

Abb. 4 Helligkeit des "White Dwarf” Sternes PG 1159-035.

2.2 Entscheidungstheoretischer Ansatz. Hier interessiert die Güte eines geschätzten Modells, gemessen mit einer Verlustfunktion. Oft ist das Ziel in einer Anwendung, eine Prognose-Güte zu maximieren.

Beispiel 2: Helligkeit des "White Dwarf" Sternes PG 1159-035

Die Daten bestehen aus 2000 Messungen von "Helligkeit" (Lichtintensitäten), welche in Abständen von jeweils 10 Sekunden gemacht wurden, siehe Abbildung 4. Interessierende Fragestellungen sind unter anderen Periodizitäten und Schwingungen der Lichtintensitäten, welche oft geeigneter im Frequenzbereich (Fourier-Bereich) analysiert werden. Der Einfachheit halber beschränken wir uns jedoch hier auf den Zeitbereich und betrachten ein stochastisches autoregressives Modell der Ordnung $p$, abgekürzt mit $A R(p)$,

$$
X_{t}=\theta_{1} X_{t-1}+\cdots+\theta_{p} X_{t-p}+\varepsilon_{t}(t=1, \ldots, n=2000),
$$

wobei $\varepsilon_{1}, \ldots, \varepsilon_{n}$ unabhängig und identisch verteilt (i.i.d.) sind mit Erwartungswert $\mathbb{E}\left[\varepsilon_{t}\right]=0$, Varianz $\operatorname{Var}\left(\varepsilon_{t}\right)=\sigma^{2}<\infty$ und $\varepsilon_{t}$ unabhängig von $\left\{X_{s} ; s<t\right\}$ ist; $\theta_{1}, \ldots, \theta_{p}$ sind die unbekannten Parameter. Natürlich weiss man a priori nicht, wie gross die Ordnung $p$ gewählt werden soll. Deshalb betrachtet man die ganze Klasse von $A R(p)$ Modellen mit $0 \leq p<\infty$,

$$
M_{A R}=\bigcup_{p=0}^{\infty}\left\{M ; M \text { ein } A R(p) \text { mit Parameter } \theta_{M} \in \Theta_{M} \subset \mathbb{R}^{\operatorname{dim}(M)}\right\} \text {. }
$$

Hierbei (und im folgenden) bezeichnet $M$ eine Modell-Struktur, $\theta_{M}$ den zur Struktur zugehörigen Parametervektor (typischerweise unbekannt) im Parameterraum $\Theta_{M}$ und $\operatorname{dim}(M)=\operatorname{dim}\left(\theta_{M}\right)$ die Dimension des Modells (der Modell-Struktur). In (2) ist 
$\operatorname{dim}(M)=p_{M}$ gerade die Ordnung des AR-Modells mit Struktur $M$. Das parametrische Modell ist dann vollständig beschrieben durch das $\operatorname{Paar}\left(M, \theta_{M}\right)$.

Die Schätzung des unbekannten $\theta_{M}$ in einer gegebenen Modell-Struktur $M$ kann hier mit der berühmten Kleinste-Quadrate Methode durchgeführt werden, welche auf Legendre und Gauss zurückgeht. Die übliche Notation dafür ist $\hat{\theta}_{M}$,

$$
\hat{\theta}_{M}=\operatorname{argmin}_{\theta_{M}=\left(\theta_{1}, \ldots, \theta_{p_{M}}\right)^{\prime}} \sum_{t=p_{M}+1}^{n}\left(X_{t}-\theta_{1} X_{t-1}-\cdots-\theta_{p_{M}} X_{t-p_{M}}\right)^{2},
$$

wobei $p_{M}$ die Ordnung der $A R$-Modell-Struktur $M$ bezeichnet. Mit dieser Schätzung konstruiert man sich eine geschätzte Prognose im Modell mit Struktur $M$ für die nächste unbekannte Zufallsvariable $X_{n+1}$,

$$
\hat{\mu}_{M ; n+1}=\hat{\theta}_{1} X_{n}+\cdots+\hat{\theta}_{p_{M}} X_{n-p_{M}+1} .
$$

Dieser gegenübergestellt steht die wahre Prognose (eines Orakels),

$$
\mu_{n+1}^{*}=\theta_{1}^{*} X_{n}+\cdots+\theta_{p^{*}}^{*} X_{n-p^{*}+1},
$$

wobei $\theta_{M}^{*}=\left(\theta_{1}^{*}, \ldots, \theta_{p^{*}}^{*}\right)^{\prime}$ den wahren Parametervektor in dem wahren $A R\left(p^{*}\right)$ bezeichnet. (Wir nehmen hier an, dass das wahre Modell in $M_{A R}$ liegt. Solch eine Restriktion wird in Kapitel 3 aufgehoben). Das Ziel ist nun, ein Risiko, zum Beispiel den erwarteten quadratischen Verlust ${ }^{2}$, zu minimieren,

$$
R(M)=\mathbb{E}\left[\left(\hat{\mu}_{M ; n+1}-\mu_{n+1}^{*}\right)^{2}\right] .
$$

Bezüglich einer solchen Risiko-Funktion definiert man die optimale Modell-Struktur in der Klasse $\mu_{A R}$ als,

$$
M_{\mathrm{opt}}=\operatorname{argmin}_{M \in \mathcal{M}_{A R}} R(M) .
$$

Das folgende erstaunliche Phänomen beschreibt jetzt aber die Andersartigkeit von wahrer und optimaler Modell-Struktur. Auch falls die wahre Struktur $M_{\text {wahr }} \in M_{A R}$, so ist im Allgemeinen

$$
M_{\mathrm{opt}} \neq M_{\mathrm{wahr}} \text {. }
$$

Die Frage nach der Optimalität einer Modell-Struktur ist also grundlegend anders als die Frage nach der wahren Struktur! Für die entscheidungstheoretische Modellwahl, oder die Modellwahl bezüglich der besten Prognose, nützt unter Umständen die Kenntnis der wahren Struktur wenig.

Wir wollen vorerst eine intuitive Erklärung für dieses, auf den ersten Blick doch paradoxe Phänomen geben. Das Risiko einer Modell-Struktur beinhaltet implizit die Schätzungen der unbekannten Parameter. Die Ungenauigkeiten bei diesen Schätzungen addieren sich

2) Es kann gezeigt werden, dass diese Risiko-Funktion auch vernünftig ist für die Schätzung der FourierTransformierten in AR-Modellen (Shibata, 1981); die interessierenden Fragestellungen im Frequenzbereich können also auch mit der hier beschriebenen Technik analysiert werden. 
mit jedem Parameter auf. Deshalb kann manchmal eine Modell-Struktur mit wenig unbekannten Parametern einer hoch-dimensionalen, wahren Struktur vorgezogen werden; obwohl man dann natürlich einen systematischen Fehler mit dem unwahren niedrigdimensionalen Modell machen wird. Diese Intuition kann mathematisch quantifiziert werden. Das Risiko für eine Struktur $M$ kann folgendermassen zerlegt werden,

$$
R(M)=\mathbb{E}\left[\left(\hat{\mu}_{M ; n+1}-\mu_{n+1}^{*}\right)^{2}\right] \approx \mathbb{E}\left[\left(\mu_{M ; n+1}^{*}-\mu_{n+1}^{*}\right)^{2}\right]+\sigma^{2} \frac{\operatorname{dim}(M)}{n},
$$

wobei $\mu_{M ; n+1}^{*}$ die beste lineare Prognose in Modell-Struktur $M$ für $X_{n+1}$ ist (bezüglich Risiko $R()$.$) . Der erste Term auf der rechten Seite der approximativen Gleichung be-$ schreibt den systematischen Fehler (Bias) zwischen der besten Prognose $\mu_{M ; n+1}^{*}$ in der Struktur $M$ (diese hat nichts mit Schätzung zu tun) und der wahren Prognose $\mu_{n+1}^{*}$, der zweite Term beschreibt den Schätzfehler, welcher sich aus den Varianzen der einzelnen Parameterschätzungen ergibt. Interessanterweise wächst dieser zweite Varianz-Term $l i$ near in der Dimension der Modell-Struktur. Insbesondere, falls $\operatorname{dim}\left(M_{\text {wahr }}\right)$ gross ist, so kann dieser für die wahre Struktur einen dominierenden Negativ-Effekt auf die PrognoseGüte (Risiko $R($.$) ) haben.$

Dieses Modellwahl-Phänomen tritt in Situationen mit allgemeinen Modellklassen $M$ und Risikofunktionen auf, siehe auch Kapitel 3 und 4. Zusammenfassend können wir also festhalten, dass die optimale Modell-Struktur ganz allgemein einen Bias-Varianz Tradeoff berücksichtigt.

Natürlich ist unsere vorhin betrachtete Risikofunktion $R($.$) unbekannt und daher auch die$ optimale Modell-Struktur $M_{\mathrm{opt}}$. Schätzungen von $R($.$) aus den Daten sind aber bekannt,$ so zum Beispiel der "Final Prediction Error" (FPE) von Akaike (1969) oder Mallows $C_{p}$ (Mallows, 1973). Eine Modellwahl für den konkreten Datensatz in Beispiel 2 wird im nächsten Kapitel 3 mit einer allgemeineren Methode durchgeführt.

\section{Modellwahl mit Akaike's Kriterium}

Wir diskutieren hier eine noch viel universeller anwendbare Methode, um entscheidungstheoretische Modellwahl durchzuführen.

Bevor wir spezifischere Annahmen über ein Modell machen, bezeichnen wir mit $P^{*}$ die (wahre) Wahrscheinlichkeitsverteilung der Daten $X_{1}, \ldots, X_{n}$. Der Werteraum von $X_{t}$ sei $\mathscr{X}(t=1, \ldots, n)$, zum Beispiel $\mathscr{X}=\mathbb{R}$. Man möchte dieses wahre $P^{*}$ schätzen, so dass man die gesamte stochastische Kenntnis zumindest approximativ besitzt.

Dazu benützt man häufig eine möglichst geeignete parametrische Modellklasse,

$$
\begin{aligned}
& \mathcal{M} \text { eine diskrete Menge, } \\
& \mathscr{P}=\bigcup_{M \in \mathcal{M}}\left\{P_{\theta_{M}}, \theta_{M} \in \Theta_{M} \subset \mathbb{R}^{\operatorname{dim}(M)}\right\} .
\end{aligned}
$$

Die Menge $\mathcal{M}$ besitzt als Elemente alle interessierenden Modell-Strukturen $M, \mathscr{P}$ ist dann die zugehörige Klasse von Wahrscheinlichkeitsverteilungen $P_{\theta_{M}}$, indiziert mit einem unbekannten Parameter $\theta_{M}(M \in \mathcal{M})$. Beispiele dafür sind die Klasse in (2), aber auch 
die Klasse in (6) in Kapitel 4, welche kategorielle Daten beschreibt. Letztere ist auch ein Beispiel dafür, wo das in Kapitel 2.2 vorgestellte Risiko $R($.$) mit dem erwarteten$ quadratischen Verlust keinen Sinn macht, da bei kategoriellen Daten keine Ordnung vorhanden ist.

Die Schätzung des unbekannten Parameter-Vektors $\theta_{M}$ in Modell-Struktur $M$ kann im Allgemeinen mit der Maximum-Likelihood Methode ausgeführt werden,

$$
\hat{\theta}_{M}=\operatorname{argmin}_{\theta_{M} \in \Theta_{M}}-\log \left(d P_{\theta_{M}}\left(X_{1}, \ldots, X_{n}\right)\right),
$$

wobei $d P_{\theta_{M}}\left(X_{1}, \ldots, X_{n}\right)$ die Wahrscheinlichkeitsdichte (oder Wahrscheinlichkeit) der Daten $X_{1}, \ldots, X_{n}$ im Modell mit Struktur $M$ und Parametervektor $\theta_{M}$ bezeichnet. Der Maximum-Likelihood Schätzer $\hat{\theta}_{M}$ gibt also im Modell mit Struktur $M$ maximale Wahrscheinlichkeit für die beobachteten Daten. Falls das Modell $P_{\theta_{M}}$ für unabhängige und identisch normalverteilte Zufallsvariablen steht, so ist der Maximum-Likelihood Schätzer gleich dem Kleinste-Quadrate Schätzer.

Wie bereits oben erwähnt möchten wir eine Risikofunktion, welche universeller anwendbar ist als diejenige von Kapitel 2.2 basierend auf quadratischem Verlust. Wir betrachten hier die sogenannte Kullback-Leibler Information für eine Modell-Struktur $M$,

$$
\operatorname{KLI}(M)=I_{n}\left(P^{*}, P_{\hat{\theta}_{M}}\right)=\int_{\mathscr{X}^{n}} \log \left(\frac{d P^{*}(x)}{d P_{\hat{\theta}_{M}}(x)}\right) d P^{*}(x) .
$$

Dieses Risiko ist auch bekannt als relative Entropie von $d P^{*} / d P_{\hat{\theta}_{M}}$ bez. $d P_{\hat{\theta}_{M}}$. Eine alternative, mehr wahrscheinlichkeitstheoretische Form dafür ist,

$$
\operatorname{KLI}(M)=C+\mathbb{E} y\left[-\log \left(d P_{\hat{\theta}_{M}}\left(Y_{1}, \ldots, Y_{n}\right)\right)\right],
$$

wobei $C=\mathbb{E} y\left[\log \left(d P^{*}\left(Y_{1}, \ldots, Y_{n}\right)\right)\right]$ eine Konstante bezüglich Modellwahl ist (keine funktionelle Abhängigkeit von $M$ ); und $Y_{1}, \ldots, Y_{n}$ sind Zufallsvariablen, welche unabhängig von den Daten $X_{1}, \ldots, X_{n}$ sind, jedoch dieselbe Wahrscheinlichkeitsverteilung $P^{*}$ haben. Diese Variablen $Y_{1}, \ldots, Y_{n}$ können als sogenannter "Test-Set" interpretiert werden: die aus den Daten $X_{1}, \ldots, X_{n}$ geschätzte Verteilung $P_{\hat{\theta}_{M}}$ wird an den neuen, von den Daten unabhängigen, Test-Variablen $Y_{1}, \ldots, Y_{n}$ evaluiert. Minimierung von $\operatorname{KLI}(M)$ bezüglich $M$ ist äquivalent zu Minimierung von $\mathbb{E}\left[-\log \left(d P_{\hat{\theta}_{M}}\left(Y_{1}, \ldots, Y_{n}\right)\right)\right]$, der geschätzten negativen log-likelihood, evaluiert am und danach gemittelt über den "TestSet".

Analog zu Kapitel 2.2 definieren wir die optimale Modell-Struktur,

$$
M_{\text {opt }}=\operatorname{argmin}_{M \in M} \operatorname{KLI}(M) .
$$

Auch hier gilt im Allgemeinen $M_{\mathrm{opt}} \neq M_{\text {wahr }}$. Die Erklärung dafür liefert wiederum eine Bias-Varianz Zerlegung,

$$
\operatorname{KLI}(M)=I_{n}\left(P^{*}, P_{\theta_{M}^{*}}\right)+\int_{\mathscr{X}^{n}} \log \left(\frac{d P_{\theta_{M}^{*}}(x)}{d P_{\hat{\theta}_{M}}(x)}\right) d P^{*}(x),
$$




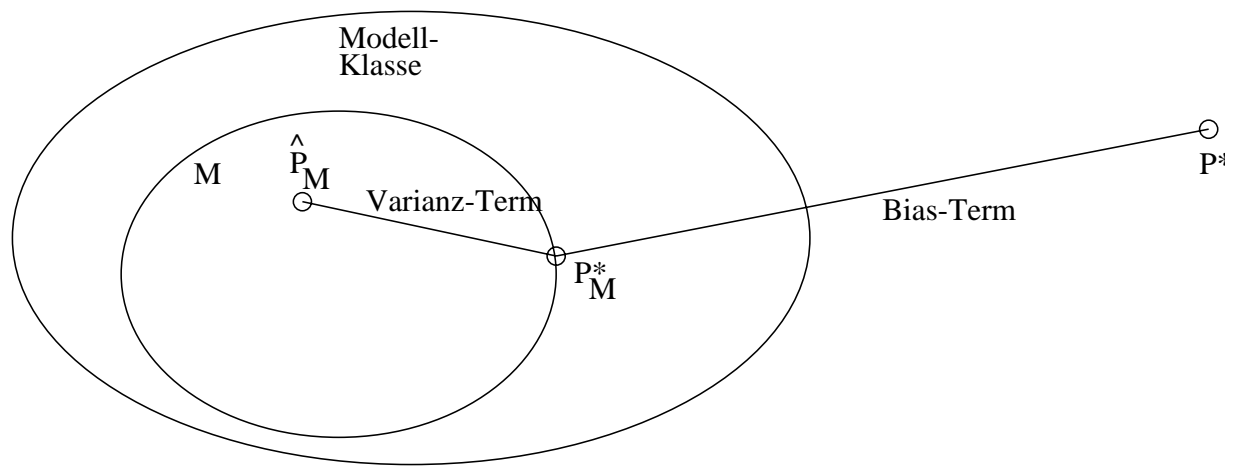

Abb. 5 Bias-Varianz Zerlegung der Kullback-Leibler Information $I_{n}\left(P^{*}, P_{\hat{\theta}_{M}}\right): \hat{P}_{M}$ und $P_{M}^{*}$ sind dabei Abkürzungen für $P_{\hat{\theta}_{M}}$, respektive $P_{\theta_{M}^{*}}$.

und unter Regularitätsbedingungen,

$$
\operatorname{KLI}(M) \approx I_{n}\left(P^{*}, P_{\theta_{M}^{*}}\right)+\frac{1}{2} \operatorname{dim}(M) .
$$

Hier ist $P_{\theta_{M}^{*}}$ die Wahrscheinlichkeitsverteilung, welche zur Struktur $M$ gehört und am nächsten zu $P^{*}$ ist (bezüglich $\operatorname{KLI}($.$) ), das heisst \theta_{M}^{*}=\operatorname{argmin}_{\theta_{M} \in \Theta_{M}} I_{n}\left(P^{*}, P_{\theta_{M}}\right)$. Die Illustration in Abbildung 5 schematisiert die Formel.

Der erste Term auf der rechten Seite von (5) beschreibt den Bias (systematischer Fehler), der zweite den Varianz-Term (verursacht durch die Schätzung von unbekannten Parametern), welcher linear in der Anzahl Parameter wächst. Falls $M$ "komplexer" wird, das heisst in einer aufsteigenden Folge $M_{1} \prec M_{2} \prec \ldots\left(M_{i} \prec M_{i+1}\right.$ bedeutet $M_{i}$ ist Untermodell von $M_{i+1}$ ) mit wachsender Anzahl Parameter, wird der Bias-Term kleiner. Die wahre Wahrscheinlichkeitsverteilung der Daten $P^{*}$ ist nicht notwendigerweise ein Element der Modellklasse $\mathcal{M}$. Trotzdem, $\operatorname{KLI}(M)$ kann bis auf die irrelevante Konstante $C$ in (4) vernünftig und sehr einfach geschätzt werden. Diese Erkenntnis von Akaike (1973) gilt heute als der "Breakthrough in Statistics Nr. 19" (deLeeuw, 1991). Eine Schätzung von $2 \operatorname{KLI}(M)-2 C$ ist

$$
A I C(M)=-2 \log \left(d P_{\hat{\theta}_{M}}\left(X_{1}, \ldots, X_{n}\right)\right)+2 \operatorname{dim}(M) .
$$

Sie trägt den Namen des Erfinders (Akaike, 1973) und heisst "Akaike Information Criterion". Der erste Term auf der rechten Seite ist ein Gütemass für den sogenannten Fit des geschätzten Modells für die Daten. Im konkreten Beispiel, wo das Modell $P_{\theta_{M}}$ für $n$ unabhängige, identisch normalverteilte Zufallsvariablen steht, ist das Gütemass eine Residuenquadratsumme. Es sagt aber nichts über das Vorhersage-Potenzial eines geschätzten Modells aus; insbesondere wird mit "komplexerer" Modell-Struktur $M$ dieses Gütemass kleiner. Der zweite Term auf der rechten Seite ist ein Bestrafungsterm, welcher "komplexe" Strukturen linear in der Dimensionalität bestraft. Die Schätzung von $\operatorname{KLI}(M)$ ist also bis auf die für die Modellwahl irrelevante Konstante $C$ in (4) ein Gütemass für den Fit plus ein Bestrafungsterm. 


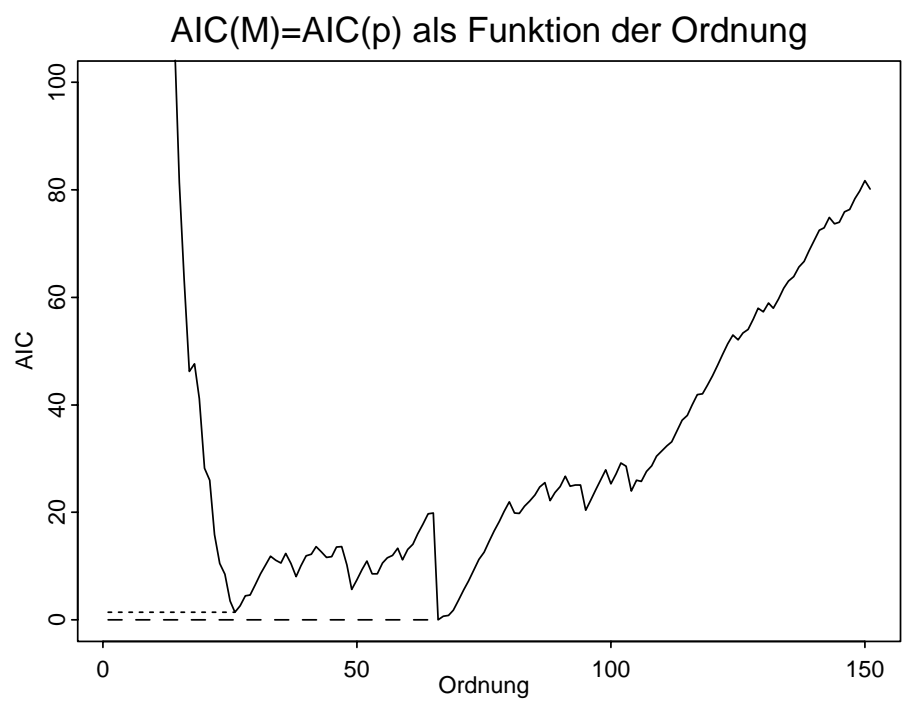

Abb. 6 AIC Kriterium für Datensatz in Beispiel 2 und Modellklasse in (2). Optimale geschätzte Ordnung ist 65 (gestrichene Linie), sub-optimale Ordnung ist 25 (gepunktete Linie).

Eine geschätzte optimale Modell-Struktur ist nun gegeben durch

$$
\hat{M}_{\mathrm{opt}}=\operatorname{argmin}_{M \in \mathcal{M}} A I C(M) \text {. }
$$

Falls die Klasse $\mathcal{M}$ von unendlicher Kardinalität ist, so restringiert man die Suche und minimiert bezüglich $M_{n}=\left\{M ; M \in \mathcal{M}, \operatorname{dim}(M) \leq c_{n}\right\}$, zum Beispiel $c_{n}=\sqrt{n}$.

Zusammenfassend halten wir fest, dass Modellwahl mit dem AIC-Kriterium universell anwendbar ist: die Kullback-Leibler Information $\operatorname{KLI}($.$) als Risikofunktion ist auch für$ nichtnormalverteilte oder kategorielle Daten sinnvoll, und das AIC-Kriterium ist auch dann eine vernünftige Risikoschätzung, falls die wahre Wahrscheinlichkeitsverteilung $P^{*}$ nicht in der betrachteten Modellklasse $M$ liegt (was realistischerweise ja der Fall sein wird).

Wir analysieren nun den Datensatz von Beispiel 2 und benützen das AIC Kriterium, um die optimale Modell-Struktur in der Klasse in (2) zu schätzen. Das Resultat ist in Abbildung 6 beschrieben. ${ }^{3)}$ Das AIC ist gross bei kleinen Ordnungen (grosser BiasTerm) und bei grossen Ordnungen (grosser Varianz-Term). Wir verfolgen nun noch kurz die sub-optimale Lösung mit Ordnung 25, da der Verlust bezüglich AIC gegenüber dem Optimum mit Ordnung 65 klein ist und einfachere Modelle bei gleicher Güte prinzipiell vorzuziehen sind. Abbildung 7 zeigt den wahren Datensatz und 8 simulierte Datensätze des Modells in (1) mit $p=25$ und normalverteilten Rauschtermen $\varepsilon_{t}$. Es ist visuell praktisch unmöglich den wahren von den simulierten Datensätzen zu unterscheiden. Dies ist eher ein Glücksfall: die Daten in Beispiel 2 lassen sich sehr gut durch ein extrem einfaches, nämlich lineares und Gauss'sches Modell beschreiben.

3) Wir nehmen dabei an, dass die Rauschterme $\varepsilon_{t}$ normalverteilt sind. 

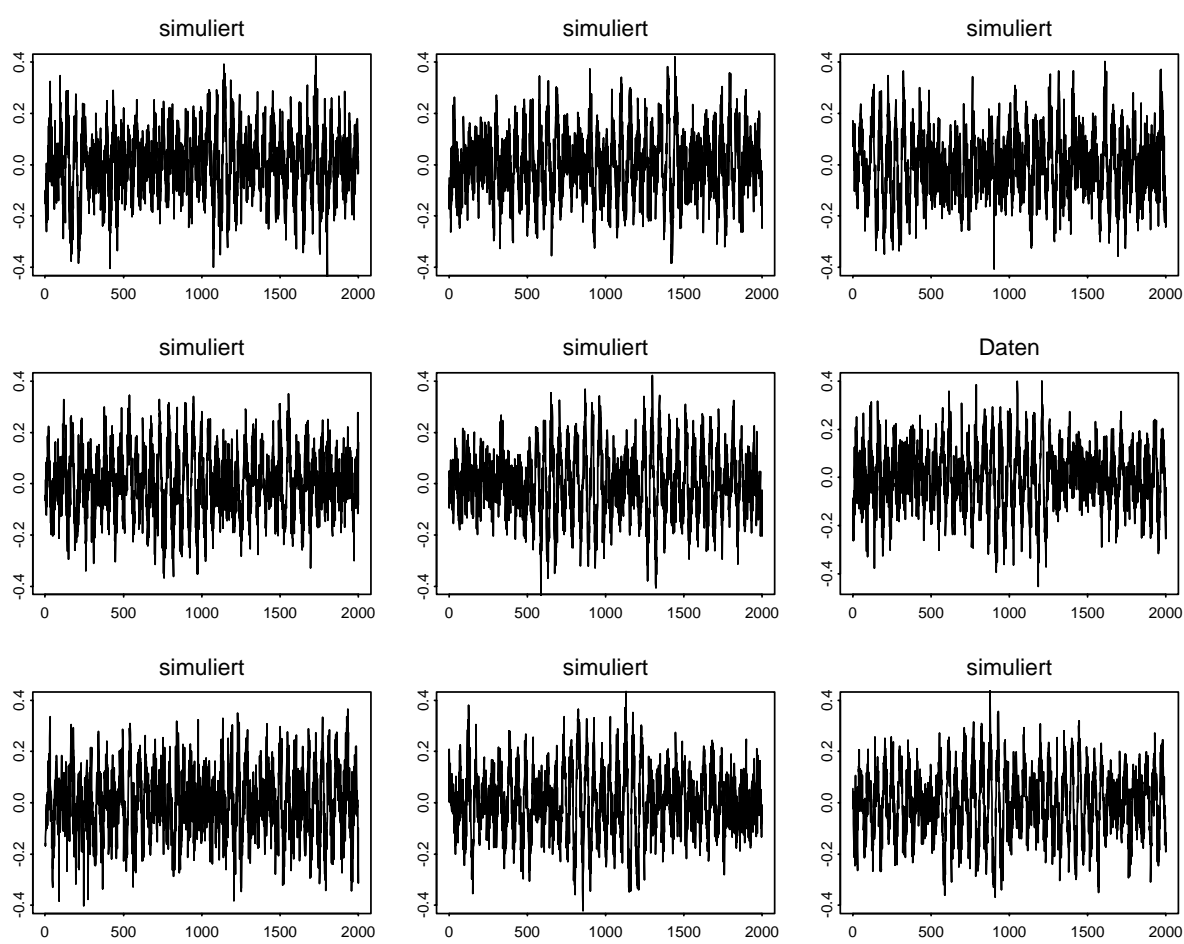

Abb. 7 Acht simulierte Datensätze und wahrer Datensatz von Beispiel 2.

\section{Vielfalt einer Modellklasse}

Wir motivieren das Thema dieses Kapitels mit einem weiteren Beispiel.

Beispiel 3: Ein-dimensionale DNA von Drosophila.

Abbildung 8 zeigt einen Ausschnitt einer DNA-Sequenz von Drosophila, welche 25000 Zeichen lang ist.

Interessierende Fragestellungen sind unter anderen die Ähnlichkeit zu anderen DNASequenzen oder Lokalisierung der "kodierenden" Teilstücke in der Sequenz. Ein naheliegendes Modell ist eine stationäre Markov-Kette der Ordnung $p$ (abgekürzt mit $M C(p)$ ) mit Werteraum $\mathscr{X}=\{A, T, G, C\}$, welche durch folgende Übergangswahrscheinlichkeiten charakterisiert ist:

$\mathbb{P}\left[X_{t}=x_{t} \mid X_{t-1}=x_{t-1}, X_{t-2}=x_{t-2}, \ldots\right]=\mathbb{P}\left[X_{t}=x_{t} \mid X_{t-1}=x_{t-1}, \ldots, X_{t-p}=x_{t-p}\right]$,

für alle $x_{t-1}, x_{t-2}, \ldots$ Dabei ist $p \in \mathbb{N}$ minimal. Dem Index $t$ kommt wegen der Stationarität keine spezielle Bedeutung zu. Diese Wahrscheinlichkeiten können in einem Parametervektor $\theta_{M} \in(0,1)^{\operatorname{dim}(M)}$ der Dimension $\operatorname{dim}(M)=3 \cdot 4^{p}$ zusammengefasst werden. Bei unbekannter Ordnung $p$ betrachtet man oft die dazugehörende Modellklasse,

$$
\mu_{M C}=\bigcup_{p=0}^{\infty}\left\{M: M \text { eine } M C(p) \text { mit Übergangsw.'keit } \theta_{M} \in(0,1)^{\operatorname{dim}(M)}\right\}
$$




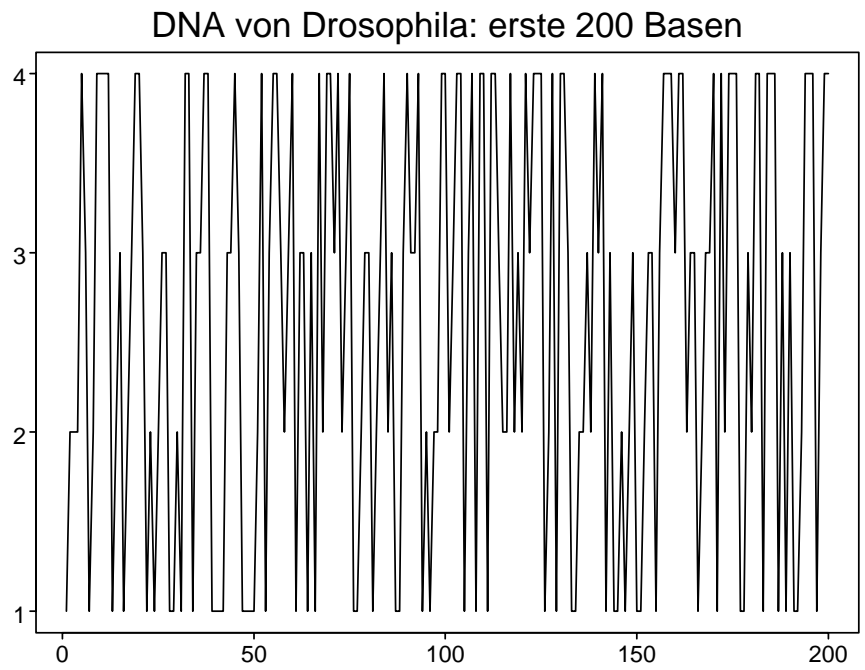

Abb. 8 DNA von Drosophila. Die Zahlen 1, 2, 3, 4 entsprechen den Basen A, T, G, C.

Obwohl diese probabilistisch eine sehr schöne und natürliche Klasse bildet, ist sie in statistischem Sinne zu simpel und strukturell zu wenig reichhaltig.

Um dies zu illustrieren, betrachten wir die Dimension (Anzahl Parameter) von Struktur $M$ als Funktion der Ordnung $p=p_{M}$, das heisst die Funktion $\operatorname{Dim}(p)=3 \cdot 4^{p}$.

\begin{tabular}{c|c|c|c|c|c|c|c}
\hline$p$ & 0 & 1 & 2 & 3 & 4 & 5 & 10 \\
\hline $\operatorname{Dim}$ & 3 & 12 & 48 & 192 & 768 & 3072 & $\approx 3.1 \cdot 10^{6}$
\end{tabular}

Dieser Tabelle entnimmt man, dass es keine "Zwischen-Modelle" gibt: die Dimension vervierfacht sich bei jeder zusätzlichen Ordnung und es gibt somit nur sehr "sprunghafte" Erhöhungen der Dimension. Dies impliziert insbesondere auch, dass man oft zu wenig Flexibilität hat, um den vorher diskutierten Bias-Varianz Trade-off gut zu berücksichtigen. Überdies hat man sehr schnell (zu) viele Parameter, um Abhängigkeiten der Ordnung $p>3$ zu modellieren. Solches wird auch in Braun \& Müller (1998) bei der statistischen Analyse von DNA-Sequenzen diskutiert.

Einen erfolgreichen Ausweg aus dieser zu simplen Modellklasse bilden die sogenannten Variable Length Markov Chains (VLMC), welche wohl zuerst in der Informationstheorie Fuss gefasst haben. Die Idee dabei ist, dass eine stationäre VLMC ein Gedächtnis von variabler Länge hat. Eine $V L M C(p)$ ist charakterisiert durch die folgenden Übergangswahrscheinlichkeiten:

$$
\begin{aligned}
& \mathbb{P}\left[X_{t}=x_{t} \mid X_{t-1}=x_{t-1}, X_{t-2}=x_{t-2}, \ldots\right]=\mathbb{P}\left[X_{t}=x_{t} \mid X_{t-1}=x_{t-1}, \ldots, X_{t-\ell}=x_{t-\ell}\right] \\
& 0 \leq \ell=\ell\left(x_{t-1}, x_{t-2}, \ldots\right) \leq p, p \in \mathbb{N} \text { minimal }
\end{aligned}
$$

für alle $x_{t-1}, x_{t-2}, \ldots$ Das Gedächtnis weist eine variable Länge $\ell$ auf, welche selbst eine Funktion der Vergangenheit $x_{t-1}, x_{t-2}, \ldots$ ist. Wiederum kommt wegen der Stationarität 

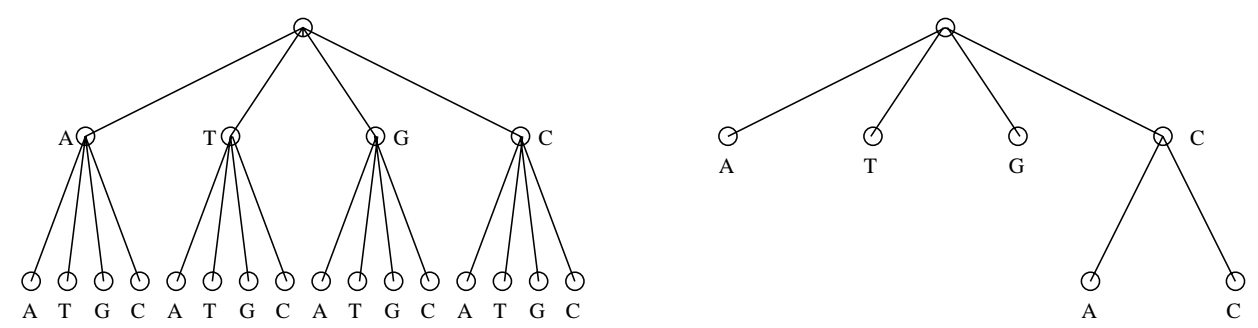

Abb. 9 Baum-Darstellung von VLMC-Gedächtnis.

dem Index $t$ keine spezielle Bedeutung zu. Ein solches Gedächtnis von variabler Länge, oder äquivalent die Funktion $\ell($.$) , kann als Baum dargestellt werden.$

Auf der linken Seite in Abbildung 9 ist das Gedächtnis einer vollen MC(2) dargestellt mit $4^{2}=16$ Zuständen (Endknoten). Rechts in Abbildung 9 das Gedächtnis einer $V L M C(2)$ mit bloss 6 Zuständen: die Vergangenheiten $X_{t-1}=A ; X_{t-1}=T ; X_{t-1}=G$; $X_{t-1}=C$ und $X_{t-2}=A ; X_{t-1}=C$ und $X_{t-2} \in\{T, G\} ; X_{t-1}=C$ und $X_{t-2}=C$ (Repräsentation mit 5 Endknoten und einem inneren Knoten). Das variable Gedächtnis liest sich wie folgt: $\ell(A, \ldots)=\ell(T, \ldots)=\ell(G, \ldots)=1, \ell(C, A, \ldots)=2$, $\ell(C, T, \ldots)=\ell(C, G, \ldots)=1, \ell(C, C, \ldots)=2$.

Mit Hilfe der obigen Baum-Darstellungen wird schnell einmal klar, dass die Klasse der VLMC's gute "Zwischen-Modelle" hat und dass gewisse lange Abhängigkeiten mit wenig Zuständen modelliert werden können; bei beidem sind Bäume mit dünnen Ästen gefragt. Die Klasse aller VLMC's besitzt also, im Gegensatz zu $M_{M C}$ in (6), eine grosse Vielfalt oder Reichhaltigkeit.

Dabei hat man sich aber ein beträchtliches Problem eingehandelt: die Klasse ist enorm gross. So ist zum Beispiel die Anzahl VLMC Untermodelle von MC(p) gleich $11\left(2^{4^{p-1}}+\right.$ $\left.\sum_{k=1}^{p-2}\left(2^{4^{k}}-1\right)\right)+1$. Die folgende Tabelle verdeutlicht die astronomischen Grössen.

\begin{tabular}{c|c|c|c|c}
\hline$p$ & 1 & 2 & 3 & 4 \\
\hline \# Untermod. & 12 & 176 & 721062 & $\approx 2.0 \cdot 10^{20}$
\end{tabular}

Auch für die modernsten Computer der nächsten Generationen sind diese Zahlen zu gross. Falls man zum Beispiel für die Berechnung eines Modells eine Sekunde Rechenzeit brauchen würde, so müsste man für alle Untermodelle bei $p=3$ bereits ungefähr 8.3 Tage und bei $p=4$ ungefähr $6.4 \cdot 10^{10}$ Jahrtausende rechnen! Eine globale Suche nach Optimalität wie zum Beispiel in Kapitel 3 mit $\hat{M}_{\mathrm{opt}}=\operatorname{argmin}_{M} A I C(M)$ ist für $p>2$ nicht mehr möglich.

Eine grosse Innovation von Rissanen (1983) hilft aber diesem Problem ab. Man kann eine Suche nach der Modell-Struktur "lokal" statt "global" durchführen. Die Idee dabei ist, eine Entscheidung für oder gegen ein Untermodell mittels Inspektion an einzelnen Endknoten von Bäumen zu machen. Dies kann in dem sogenannten Context-Algorithmus implementiert werden, welcher mit $O(n \log (n))$ wesentlichen Operationen arbeitet. Neuere Resultate zeigen, dass dieser Context-Algorithmus asymptotisch den wahren unterliegenden minimalen Zustandsraum (Baum) findet und statistisch effizient (asymptotisch optimal) ist. 


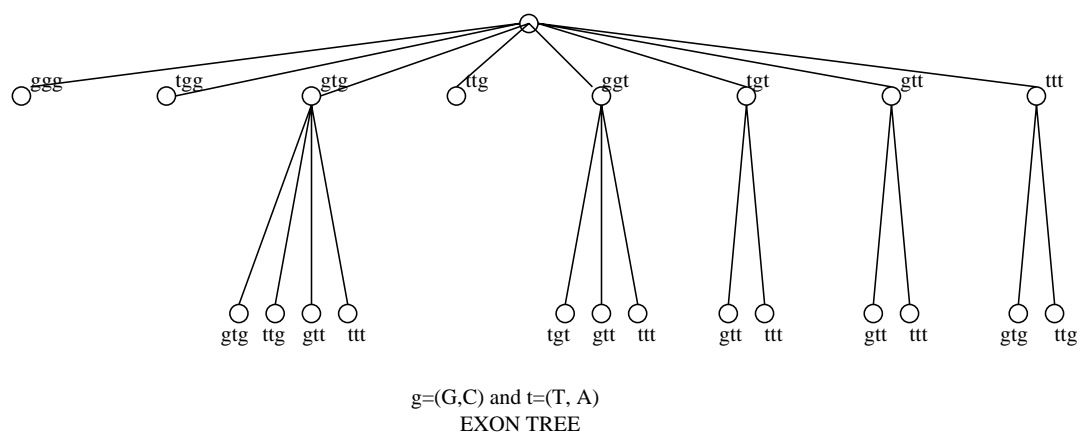

Abb. 10 Triplet-Baum Darstellung von Gedächtnis einer angepassten VLMC für binärisierte DNA-Sequenz in Beispiel 3.4)

Wir analysieren nun damit den DNA-Datensatz von Beispiel 3. Aus molekularbiologischen Gründen wird oft eine binäre Sequenz gebildet. Wir identifizieren wie folgt: $g=(G, C), t=(T, A)$. Alle in diesem Kapitel gemachten Überlegungen machen wir jetzt für den binären Fall. Eine binäre VLMC wird mit dem Context-Algorithmus auf dem Exon-Teil $\left.{ }^{4}\right)$ der Sequenz (25000 Basen) angepasst. Das angepasste VLMC-Modell ist von Ordnung 6 und hat 26 Parameter. Interessanterweise kommen im Baum bloss Äste der Länge 0,3 und 6 vor: man kann also eine Darstellung in Triplets geben, siehe Abbildung 10.5) Diese Darstellung ist interessant, weil man von der Molekularbiologie weiss, dass Aminosäuren von Triplets der DNA kodiert werden. Diese Triplet Baum-Darstellung hat also eine schöne Interpretation in der Molekularbiologie. Obschon die Modellwahl mit dem Context-Algorithmus primär darauf abzielt, gute Vorhersage-Modelle zu finden, haben wir es hier mit einem Beispiel zu tun, bei dem ein gewähltes Modell zusätzlich eine sehr schöne strukturelle Interpretation hat. Es ist also ein Beispiel, wo möglicherweise $M_{\mathrm{opt}} \approx M_{\mathrm{wahr}}$, was gemäss dem früher formulierten Modellwahl-Phänomen nicht die Regel ist.

Die Idee von VLMC's kann auch mit einigem Aufwand auf das schwierigere Problem von stationären $\mathbb{R}$-wertigen Zeitreihen übertragen werden, wir nennen diese Modelle dann verallgemeinerte VLMC's. Analog zu vorhin erhält man auch in diesem Falle eine vielfältige Modellklasse. Wir illustrieren nochmals an einem Beispiel.

Beispiel 4: Tägliche Returns von BMW Aktien.

Abbildung 11 zeigt 1000 tägliche Return-Daten des BMW Aktienpreises, das heisst $X_{t}=\log \left(P_{t} / P_{t-1}\right)$ mit dem Aktienpreis $P_{t}$. Die interessierende Fragestellung ist oft direkt von mathematischer Natur: die Wahrscheinlichkeitsverteilung eines Returns der Zukunft, gegeben die Werte von heute und der Vergangenheit. Kenntnis davon ermöglicht die Konstruktion von diversen Risiko-Massen im sogenannten Risk-Management.

4) Dies ist der Teil der DNA-Sequenz, wo man a-priori weiss, dass er "kodiert".

5) Zum Beispiel beschreibt der zweite Knoten von links in Tiefe 2 die Übergangswahrscheinlichkeiten als $\mathbb{P}\left[X_{t}=x_{t} \mid\left(X_{t-1}, \ldots, X_{t-6}, \ldots\right)=(g, t, g, t, t, g, \ldots)\right]=\mathbb{P}\left[X_{t}=x_{t} \mid\left(X_{t-1}, \ldots, X_{t-6}\right)=(g, t, g, t, t, g)\right]$. 


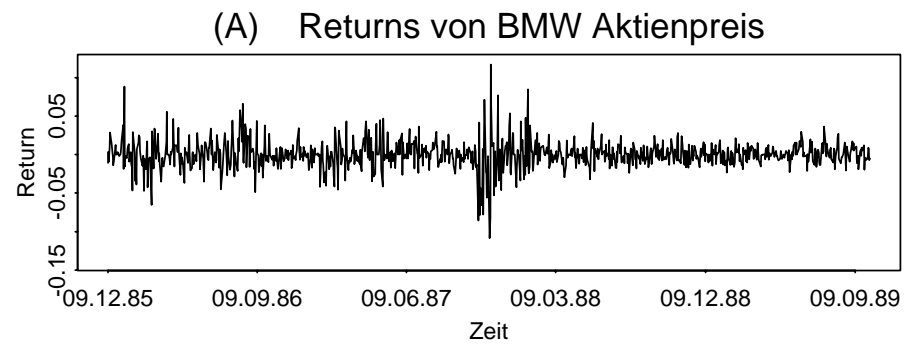

(B)

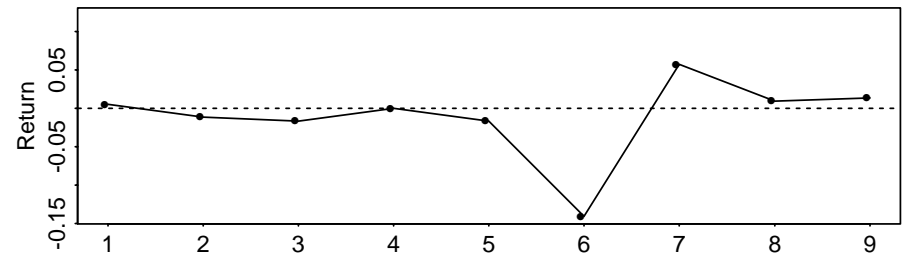

Abb. 11 (A): 1000 tägliche Returns. (B): Die nächsten neun täglichen Returns der darauffolgenden Tage. Tag 6 ist der Montag nach dem Wiedervereinigungs-Wochenende in Deutschland.
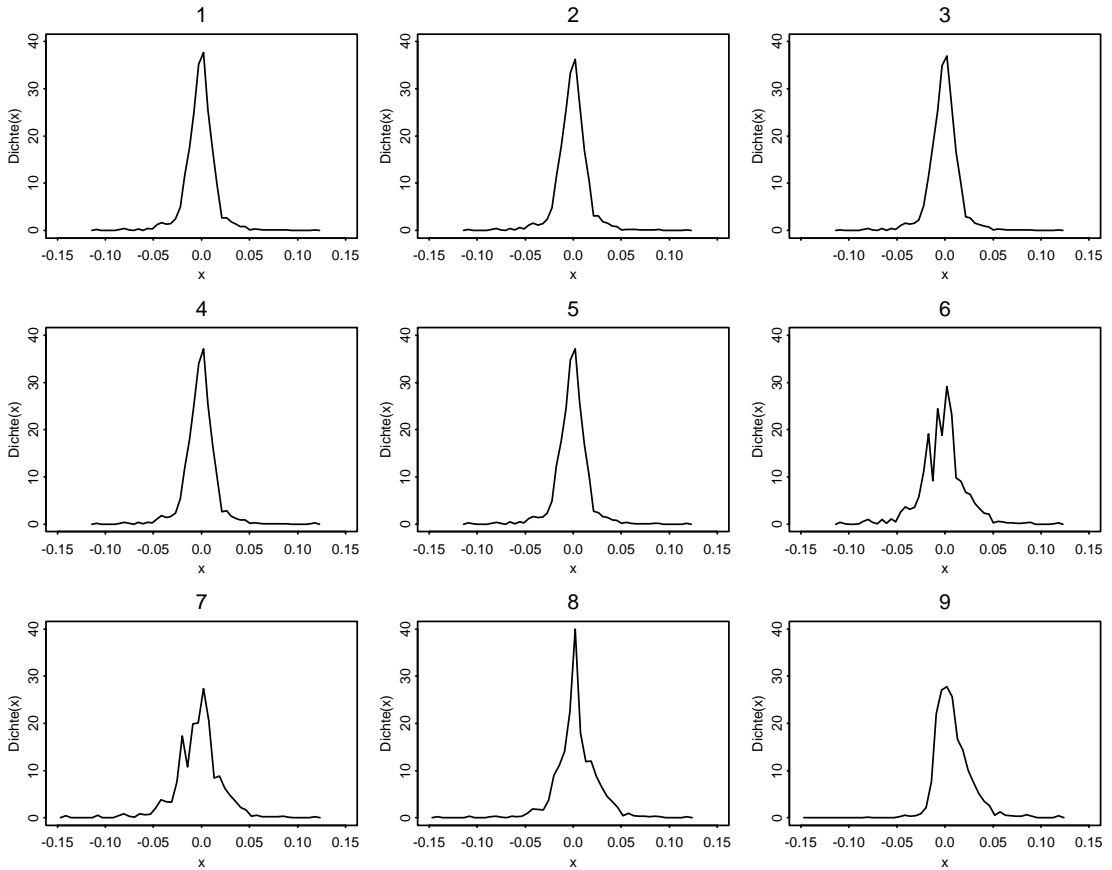

Abb. 12 1-Tage-Vorhersagedichten für die neun Tage in Abbildung 11 (B). 
Für eine angepasste verallgemeinerte VLMC zeigt Abbildung 12 die 1-Tage-Vorhersagedichten für die 9 zukünftigen Tage von Abbildung 11 (B): Das dabei benützte Modell hat 65 Parameter, was viel mehr Komplexität aufweist als die für Finanzzeitreihen oft benützten GARCH-Modelle. Die grossen und kleinen Returns an den Tagen 6 und 7 werden im Sinne von grosser Varianz bei den Vorhersagedichten korrekt prognostiziert. Die Vorzeichen der Returns können (natürlich) nicht vernünftig vorhergesagt werden. Erstaunlicherweise werden aber die "Switches im Regime" an den Tagen 6 und 8 richtig vorhergesagt. Wie man solche "Switches im Regime" quantitativ aus den Daten geschätzt hat, kann zum Beispiel folgendermassen formuliert werden. Man beobachtet eine relative bedingte Häufigkeit von $11.1 \%$ für das Unterschreiten des 5\%-Quantils der gesamten Reihe, gegeben die Vergangenheit ähnlich wie bei Tag 6 (die Ähnlichkeit ist mit Hilfe des benützten Modells definiert). Dies entspricht mehr als einer Verdoppelung zum unabhängigen Fall, wo zukünftige Werte nicht von der Vergangenheit abhängen. Fairerweise fügen wir an, dass verallgemeinerte $V L M C$-Vorhersage für "Switches im Regime" nicht immer so phantastisch wie in diesem Beispiel funktioniert.

\section{Komplexe stochastische Modelle}

In Vorhersage-Problemen werden öfters komplexe stochastische Modelle verwendet wie Neuronale Netze, "Finite-State Machines" oder auch Methoden aus dem Gebiet von Pattern Recognition. Die in Kapitel 4 vorgestellten VLMC's sind Spezialfälle von "FiniteState Machines", welche vor allem in der Informationstheorie entwickelt wurden. In allen Fällen konstruiert man eine (für die entsprechende Anwendung) vielfältige Modellklasse. Das Finden von passenden komplexen, stochastischen Prognose-Modellen ist dann äquivalent zum Problem der entscheidungstheoretischen Modellwahl in einer Klasse wie in (3). Insbesondere müssen zunehmend komplexe Modelle stärker bestraft werden. Wie wir in Kapitel 4 gesehen haben, kann man bei vielfältigen Modell-Klassen aus rechentechnischen Gründen keine globale Modell-Struktur Suche mit zum Beispiel dem AIC Kriterium durchführen. Der Context-Algorithmus (Rissanen, 1983), welcher in Kapitel 4 erwähnt wurde, erweist sich im Falle von VLMC's als ein vernünftiges Verfahren, welches lokal sucht. In diesem Spezialfall vereinfachen der endliche Werteraum der Variablen $X_{t}$ und die hierarchische Struktur in der Modell-Klasse, nämlich dass das Gedächtnis einer VLMC immer noch aus zeitlich aufeinanderfolgenden Variablen besteht, das Problem beträchtlich. Viel schwieriger wird es bei Werteräumen wie $\mathbb{R}^{d}$ mit $1 \leq d<\infty$, zum Beispiel bei den in Beispiel 4 verwendeten verallgemeinerten VLMC's, und bei nicht-hierarchischen Modellen.

Wir möchten nun noch mit einer etwas erweiternden und ausblickenden Sicht schliessen. In der angewandten Statistik betrachtet man oft, insbesondere bei komplexen Problemen, mehrere "gute" Modelle, um vielleicht ein vollständigeres Bild zu erhalten. Warum überhaupt das optimale Modell? Interessanterweise wird auch diese Frage bei reinen "BlackBox" Verfahren und komplexen stochastischen Vorhersage-Systemen wieder aufgegriffen. Rein experimentell ist eine beträchtliche Evidenz vorhanden, dass Mittelbildung über mehrere Prognosen in verschiedenen komplexen Modellen letztendlich eine bessere Vorhersage liefert. Solche gemittelten Prognosen lassen sich sehr einfach mit Hilfe von zufälliger Perturbation implementieren. Man nimmt die einem persönlich am meisten zusagende komplexe Modell-Klasse mit deren Schätz-Algorithmus und mittelt dann 
über Prognosen, von denen jede jeweils durch geeignet gewählte zufällige Perturbation der Daten (des Inputs des Systems) zustandegekommen ist, siehe zum Beispiel Breiman (1996). Die theoretischeren Gründe dieses simplen, aber oft effektiven Tricks sind bis heute weitgehend unerforscht.

Bemerkung. Dieser Artikel basiert auf meiner Einführungsvorlesung an der ETH Zürich vom Juni 1998. Mein spezieller Dank richtet sich an Prof. Urs Stammbach von der ETH Zürich für die Einladung zur Veröffentlichung, an Dr. Werner Stahel von der ETH Zürich für konstruktive Kommentare zum Manuskript und an Prof. David Brillinger von der University of California in Berkeley für die Aufarbeitung der Abbildungen 2 und 3. Letztere sind Modifikationen aus [5], welche hier mit der Genehmigung von John Wiley \& Sons Limited abgedruckt sind.

\section{Literatur}

[1] Akaike, H. (1969). Fitting autoregressive models for prediction. Annals of the Institute of Statistical Mathematics 21, 243-247.

[2] Akaike, H. (1973). Information theory and the maximum likelihood principle. In 2nd International Symposium on Information Theory (Eds. B.N. Petrov and F. Csàki), pp. 267-281. Akademiai Kiàdo, Budapest.

[3] Braun, J.V. \& Müller, H.-G. (1998). Statistical methods for DNA sequence. Statistical Science 13, $142-162$.

[4] Breiman, L. (1996). Bagging predictors. Machine Learning 26, 123-140.

[5] Brillinger, D.R. (1997). Random process methods and environmental data: the 1996 Hunter Lecture. Environmetrics 8, 269-281.

[6] deLeeuw, J. (1991). Introduction to Akaike (1973) Information theory and an extension of the maximum likelihood principle. In Breakthroughs in Statistics, Vol. I, Foundations and Theory (Eds. N.L. Johnson \& S. Kotz), pp. 599-609. Springer, New York.

[7] Friedman, J. \& Tibshirani, R. (1984). The Monotone Smoothing of Scatterplots. Technometrics 26, 243-250.

[8] Mallows, C.L. (1973). Some comments on $C_{p}$. Technometrics 15, 661-675.

[9] Rissanen, J. (1983). A universal data compression system. IEEE Transactions on Information Theory IT-29, 656-664.

[10] Shibata, R. (1981). An optimal autoregressive spectral estimate. Annals of Statistics 9, 300-306.

Peter Bühlmann

Seminar für Statistik

Departement Mathematik, ETH Zentrum

CH-8092 Zürich 